\title{
LA METODOLOGÍA DE RESOLUCIÓN DE PROBLEMAS Y EL DESARROLLO COGNITIVO: UN PUNTO DE VISTA POSTPIAGETIANO
}

\author{
POMÉS RUIZ, J. \\ Departamento de Química. Universidad Pública de Navarra. \\ Carretera del Sadar s/n. 31006-Pamplona.
}

\begin{abstract}
SUMMARY
In this work we studied the influence of the problem-solving methodology used on the efficiency. with which students' intellectual potential can be exploited. The paper shows the varying degree of interest of students in real problems as determined by application of straightforward exercises and describes the origin of the difficulties found by students in working out problems. Finally, the different methodologies were applied to questions on stoichiometry topics and the results obtained on analysing errors made by students in their replies testified to the validity of the thesis originally put forward.
\end{abstract}

\section{INTRODUCCIÓN}

La justificación de la educación científica en cualquier plan de estudios debe fundamentarse, hoy más que nunca, en las posibilidades de desarrollo intelectual que ofrecen las operaciones mentales exigidas por el aprendizaje de las ciencias experimentales. En nuestra cultura occidental se aprecia una tendencia acusada a valorar esos procesos intelectuales por encima de una aplicación instrumental cerrada de los contenidos. En la actualidad, no es suficiente que los alumnos conozcan los fundamentos explicativos de los fenomenos científicos y que adquieran una cultura que les permita moverse en nuestra sociedad tecnológica; en nuestros días emerge, como una exigencia preferente de la enseñanza de la ciencia, la utilización de los procesos intelectuales involucrados en su aprendizaje, para Iograr la optimización de las potencialidades intelectuales de los alumnos.

Una estrategia eficaz para favorecer ese desarrollo cognitivo es el empleo de una metodología de resolución de problemas que obligue al alumno a operar al límite de sus posibilidades intelectuales. Este método a menudo no coincide con las preferencias de hábitos de trabajo de los alumnos. Como indica Cusak (1986), éstos prefieren un adiestramiento en técnicas que les proporcionen de modo automático la respuesta a un razonamiento con procesos que impliquen innovación, descubrimiento, desequilibrio con los saberes previos, creatividad, y en definitiva, esfuerzo mental.

Algunos investigadores (Selvaratnam y Frazer 1982 , Morris, Lazonby y Waddington 1982), buscando una más asequible resolución de los problemas, han ideado técnicas que estandarizan las cuestiones científicas de química convirtiéndolas en «problemas tipo». De este modo, accediendo tácitamente a las poco exigentes demandas de los alumnos, restan importancia a la contribución de estos problemas al entrenamiento de los procesos mentales. El presente trabajo quiere contribuir al 
desarrollo de estrategias de trabajo intelectual a través de la resolución de problemas de química, para lo que parece previo un análisis de las dificultades que encuentran los alumnos.

\section{LAS DIFICULTADES DE LA RESOLU. CION DE PROBLEMAS}

Con frecuencia las dificultades encontradas por los alumnos en la resolución de problemas tienen alguna de estas causas: 1) los errores conceptuales previos detectados en los alumnos (Gil y Carrascosa 1985 y Driver 1989), y 2) la ausencia de los procesos intelectuales que exige el problema. Aunque los dos agentes se dan con frecuencia juntos, y tienen probablemente una misma raíz, este trabajo sólo desea profundizar en esa carencia del razonamiento avanzado. Para que la primera causa no se solape con la segunda-objeto de este estudio-, la investigación ex-post facto de resolución de problemas que se presenta, aplicó cuestiones en las que los conceptos científicos requeridos eran elementales para la muestra de alumnos utilizada.

$\mathrm{Niaz}$ (1989) indica que los procesos intelectuales exigidos para la comprensión de un curso de ciencia de enseñanza secundaria, precisan las siguientes habilidades:

a) Habilidad para transformar y procesar los datos en varias direcciones (es una manifestación del razonamiento formal), para obtener soluciones que impliquen un conociniento operativo.

b) Habilidad para procesar simultáneamente un gran número de hechos o pasos (etapas) en la ejecución de una tarea intelectual, destreza que Pascual-Leone (1978) denomina «M-Capacity».

c) Habilidad para separar la información relevante de la irrelevante.

d) Conocimientos previos de conceptos y hechos especí" ficos de la cuestión objeto de estudio.

Dentro de estas destrezas, este estudio desea destacar la consideración de la «M-Capacity», capacidad del aprehendiente que va estrechamente unida a la «M-Demand». La capacidad «M-Demand» es función de la dificultad del problema, y se define como el máximo número de pasos (esquemas) que el sujeto debe movilizar (activar) simultáneamente en el curso de la ejecución de ese problema determinado. Viene a ser un modo de obtención de las dimensiones esenciales del trabajo intelectual requerido por un problema, que el sujeto debe contemplar al mismo tiempo en su mente para encontrar la solucion.

Según Niaz (1988), hay una correlación entre las habilidades exigidas en la resolución de un problema y la capacidad mental de los alumnos que lo resuelven, correlación que es mayor al aumentar la «M-Demand».
Otro factor a tener en cuenta, es la independencia de campo, habilidad que permite la extracción de la información crítica que exige la resolución del problema desde un contexto y su aplicación a un marco diferente. La independencia de campo ayuda a resolver problemas, al implicar una movilidad o trasportabilidad de los conceptos de unas áreas a otras.

Acerca del papel que desempeñan los factores cognitivos en el aprendizaje de la química, Chandran Treagust y Tobin (1987) mantienen que los logros en el aprendizaje de esta materia son función de los conocimientos previos que tienen los alumnos y del grado de desarrollo formal de los mismos, por lo que es de capital importancia que los profesores conozcan la calidad de los conocimientos previos a la par que debieran organizar activi. dades, diferenciadas según el grado de desarrollo formal de los alumnos, para que todos ellos puedan entrenarse con tareas apropiadas a sus necesidades.

\section{INTERÉS DE LOS PROBLEMAS FRENTE AL DE LOS EJERCICIOS}

En ocasiones, se aplica el término «problema» a una cuestión que en realidad es un simple ejercicio, confusión que empobrece las posibilidades didácticas de ambas tareas. Así, un problema puede ser útil para una optimización de las estrategias de razonamiento, mientras que la utilidad de un ejercicio con frecuencia debe estax dirigida a esclarecer, aplicar o ejemplificar un concepto teórico.

Dado que el objeto de este trabajo se refiere exclusiva mente a problemas y no a ejercicios, me detendré breve. mente para distinguirlos. La diferencia esencial entre ejercicios y problemas viene dada por exigir estos últimos el aporte por parte del sujeto de algo nuevo, desconocido hasta entonces. Por el contrario, un ejercicio no supone sino una aplicación de lo ya conocido a un ejemplo más. Para resolver un problema, el alumno debe esforzarse en una interacción entre la pregunta y el intento individual de responder a esa pregunta, tensión mediante la cual se puede lograr que aflore una aportación nueva, desconocida al inicio.

El uso đe algoritmos y demás procedimientos rutinarios reduce la «M-Demand» de los problemas al tender a convertirlos en simples ejercicios memorísticos que no favorecen el desarrollo cognitivo del alumno. Una aportación interesante para superar los inconvenientes de los ejercicios y optimizar el aprendizaje es la transformación de aquéllos en problemas, aportación sugerida por Garret, Satterly, Gil-Pérez y Martínez-Torregrosa (1990). Para que el uso de un algoritmo no favorezca un aprendizaje mecánico, debe explicitarse la relación entre el concepto científico y el significado epistemológico del algoritmo, tarea que hace laboriosa la resolución de una cuestión y que con frecuencia se omite.

Para evitar que un problema se resuelva «a modo de ejercicion, se debe implicar a los alumnos en la resolución: no hacerlo por ellos, sino hacerlo con ellos. Este entrenamiento constituye una eficaz ayuda para cruzar el 
foso de la dificultad entre lo que ya se conoce y lo nuevo que ha de aprenderse.

\section{LA METODOLOGÍA DE LA RESOLU- CIÓN DE PROBLEMAS EN EL AULA}

En nuestra búsqueda de una metodología que ayude al desarrollo del alumno, no resulta sencillo determinar qué es lo prioritario, pues son demasiadas las variables aptitudinales y actitudinales de docentes y discentes para tenerlo en cuenta, al margen de los conocimientos y errores conceptuales previos que tiene cada alumno, lo que todavía hace más compleja la jerarquización de factores. A mi juicio, no existe una metodología perfecta aplicable a cualquier situación, si bien se pueden dar unas pautas generales que convenientemente adaptadas en función de Ias circunstancias particulares de cada situación de aprendizaje, puedan facilitar un mejor rendimiento en los procesos cognitivos de los alumnos.

La primera consideración a tener en cuenta (Bodner 1986) es la importancia de las etapas preliminares de la resolución de problemas: 1) la selección de información relevante, y 2) su reestructuración cognitiva, esto es, la armonización entre los saberes previos y los nuevos contenidos, equilibrio dinámico del que devenđrán los nuevos aprendizajes significativos.

La resolución de un problema debe comenzar por una lectura detenida y activa del enunciado de modo individualizado, en la que el alumno se dẹe hacer preguntas. En muchas ocasiones las respuestas que más ayudan a un aprendizaje significativo son las que se derivan de las propias preguntas que se hace el alumno, quien debe adquirir el hábito de autointerrogarse concienzudamente -en profundidad y durante suficiente tiempo- para encontrar el modo de llegar desde lo que sabe a lo que le pide el problema. Hayes (1980) sistematiza las tareas previas para resolver un problema en seis etapas:

1) Hallazgo del problema (reconocimiento de que existe un problema).

2) Representación del problema (comprensión del foso que hay que cruzar).

3) Planificación de la solución (escoger un método para cruzar el foso).

4) Llevar adelante el plan.

5) Evaluación de la solución (bondad del resultado).

6) Consolidación del aprendizaje obtenido desde la experiencia de la resolución de un problema.

La adquisición de estrategias estimuladoras de un auténtico aprendizaje y de procesos de alto valor cognitivo en los alumnos precisa la práctica de una metodología activa por parte de sus profesores. En efecto, losestudiantes no pueden ser más analíticos de lo que muestran ser sus docentes en una sesión de resolución de problemas. Así, con frecuencia, los profesores intentan enseñar a hacer problemas mediante la muestra de la resolución de algunos ejemplos, como si con el simple papel de espectador pasivo de mera exposición de las ecuaciones de algoritmos que dan solución al problema bastara para lograr el aprendizaje pretendido.

\section{EL DESARROLLO DE LAS OPERACIO- NES FORMALES MEDIANTE LA RESOLU- CION DE PROBLEMAS}

Aunque las operaciones formales son consideradas por algún autor (Pozo 1989) como un razonamiento ideal -cuya liberación de las influencias semánticas es difícilson indispensables como referencia en la psicología educativa. No se desea soslayar el hecho comprobado de que muchas personas no culminen a lo largo de su vida la plena adquisición de las operaciones formales, ni el que su acceso se inicie en la mayoría de la población a edades considerablemente superiores a las descritas por Piaget (Lawson 1985).

El empleo de las operaciones formales es ineludible a la hora de tratar el nivel de desarrollo intelectual. La aplicación de las teorías piagetianas a la enseñanza de las ciencias ha proporcionado resultados preocupantes, al no haber sido adquiridas las operaciones formales en su nivel elemental (3A) en un $70 \%$ de los alumnos de 15 años, y siendo estas operaciones imprescindibles para la comprensión de un curso de ciencia de enseñanza secundaria (Shayer y Adey 1981). Esta carencia del razonamiento formal y la necesidad imperiosa de su dominio mueven al empleode estrategias de desarrollo de operaciones formales, y de las habilidades que éstas albergan: «Mcapacity», reestructuración cognitiva, independencia de campo, etc.

Para que una cuestión se pueda categorizar de exigencia de operaciones formales, se requiere que la misma exija un razonamiento hipotético-deductivo, es decir, un análisis y comparación de varias combinaciones y posibilidades. Por ejemplo, el balance de una ecuación puede requerir pensamiento formal si el alumno necesita establecer una hipótesis. Por ello, con frecuencia, el ajuste de una ecuación por el método del ión-electrón exige pensamiento formal, mientras que hacerlo por el número de valencia puede no requerir sino operaciones concretas.

\section{APLICACIÓN A PROBLEMAS ESTE- QUIOMÉTRICOS: ANALISIS DE UNA IN- VESTIGACIÓN EX-POST FACTO}

Son varios los autores -Poole (1989), Kramers-Pals y Pilot (1988) entre otros- que presentan métodos para transformar un problema đe estequiometría en una aplicación mecánica de procedimientos rutinarios «automáticos», mediante el empleo de diversas técnicas que recuerdan en algún caso una metodología conductista. Otro proce- 
dimiento es la resolución de un probiema estequiométrico (p.e., de balance de materia) por factores de conversion, procedimiento que, al igual que los anteriores, puede suponer una reducción de la potencialidad de desarrollo cognitivo y un empobrecimiento del aprendizaje significativo descrito por Ausubel (1983).

Un modo de detectar la influencia de las habilidades cognitivas descritas, y más en concreto la «M-capacity», es el ofrecido por el análisis de las respuestas a problemas distintos a los resueltos en el aula, para evitar que el alumno conteste mediante una sustitución mecánica del correspondiente algoritmo matemático previamente memorizado. Para apreciar el efecto de las tesis aquí mantenidas, se propusieron tres problemas de estequiometría que satisfacían los requerimientos de esta investigación, a 150 alumnos de Química General de un primer curso de una Escuela de Ingenieros. Dichos problemas hacian referencia a la reacción entre los componentes de una mezcla de gases; son los siguientes:

1. Se queman $70 \mathrm{~cm}^{3}$ de una mezcla gaseosa de metanol $\mathrm{CH}_{3} \mathrm{OH}$ y etanol $\mathrm{C}_{2} \mathrm{H}_{5} \mathrm{OH}$ con la cantidad justa de aire para que la reacción sea completa, obteniéndose $\mathrm{CO}_{2}(\mathrm{~g})$ y $600 \mathrm{~cm}^{3}$ de $\mathrm{N}_{2}$ (g). La composición volumétrica del aire es del $80 \%$ en nitrógeno y $20 \%$ en oxígeno. Calcúlese la composición volumétrica de la mezcla inicial y el volumen de cada gas obtenido.

2. La combustión completa, con oxígeno puro en exceso, de una mezcla de metanol $\mathrm{CH}_{3} \mathrm{OH}$ y etanol $\mathrm{C}_{2} \mathrm{H}_{5} \mathrm{OH}$ dio una mezcla que, tras eliminat el oxígeno sobrante, contenía en volumen un $36,84 \%$ de dióxido de carbono y el resto vapor de agua. Calcular el porcentaje en volumen de metanol en la mezcla inicial.

En ambos ejemplos se observó que un $52 \%$ de los alumnos cometía el mismo error: suponer que la mezcla de gases era equimolecular, un mol de cada alcohol en la mezcla inicial. Partiendo de ese error, Ia totalidad de los alumnos intentó, y la mayoría lo consiguió satisfactoriamente, ajustar de modo cuidadoso la combustión de una mezcla equimolecular, sin percatarse de que, de acuerdo con el enunciado, ésta era la pregunta que hacía el problema, y por ello no podian partir de la respuesta.

¿Cuál puede sex la razón de este error típico? No parece aventurado conjeturar que, bajo los errores detectados, subyace una ausencia de la «M-capacity», manifiesta en Ia dificultad de discemir y procesar simultáneamente todas las variables relevantes: ajuste estequiométrico de la combustión de los alcoholes y la proporción en que estas sustancias se encuentran. El alumno suele ajustar una única ecuación con un mol de cada reaccionante y no contempla su proporción: esto es, no realiza disociación de variables.

La misma causa puede ser la responsable del énfasis puesto por el alumno en el ajuste de una reacción química y su olvido de que la reacción, al ser de equilibrio, no es completa, y que por consiguiente al final quedan reaccionantes. Otro efecto que dificulta la resolución de problemas es un «operativismo mecánico» (Gil Pérez, Martínez Torregrosa y Senent Pérez 1988), que conduce a operar con las cifras lo antes posible, sin que ello vaya precedido de un razonamiento consistente con esos cálculos. Algo similar se detecta en la resolución del ejemplo 3:

3. A una temperatura dada, la reacción $\mathrm{Xe}(\mathrm{g})+2 \mathrm{~F}_{2}(\mathrm{~g})<==\Rightarrow$ $\mathrm{XeF}_{4}(\mathrm{~g})$ da Iugar a un $40 \%$ de $\mathrm{XeF}_{4}$ comenzando con 0,50 moles de $\mathrm{Xe}$ y 0,80 moles de $\mathrm{F}_{2}$ en un recipiente de dos litros. Calcular el valor de $\mathrm{K}_{c}$ a esa temperatura. ¿Cuántos moles adicionales de $\mathrm{F}_{2}$ deben añadirse para que la conversión sea al $80 \%$ ? (Los porcentajes se refieren a ia reacción completa, estoes a aquélla en que se ha consumido alguno o los dos reaccionantes).

Un $48 \%$ de los alumnos interpretó que la reacción era completa; un buen número $(30 \%)$ ignoró cuál era el reactivo limitante sobre el que había que calcular el porcentaje de transcurso de la reacción, etc. La razón general de la dificultad que representa para el alumno la detección de las distintas variables y su procesamiento simultáneo no es sino disponer de una baja capacidad de abstracción para ver más allá del simple problema concreto del ajuste de una reacción química. A su vez, encierra una deficiencia en las estrategias de resolución de problemas: la falta de entrenamiento para buscar, en primer lugar, todas las variables que encierra el problema $y$, después, ponerse a resolverlo.

\section{CONCLUSIONES}

Enumeraré de modo conciso las estrategias cognitivas deducibles de este artículo, con el fin de facilitar su aplicación en el aula. La idea central que sugiere este artículo es mostrar el interés que tiene el entrenamiento en estrategias intelectuales que favorezcan el avance del desarrollo intelectual; así, el ejercicio de una correcta metodología de resolución de problemas constituye un poderoso y eficiente utensilio para conseguir las habilidades que involucra la intelección de un curso de ciencia, habilidades que son manifestaciones esenciales de la capacidad más general que representa el razonamiento formal.

1. Conviene percartarse, con anterioridad a una sesión de problemas, de la calidad de los conceptos previos imprescindibles para abordar el problema en cuestión.

2. Habituar a los alumnos a una lectura interrogativa de los enunciados, para favorecer con sus propias preguntas el descubrimiento de la información relevante contenida en el problema.

3. De ordinario el profesor deberá conducir la resolución de problemas con una metodología activa, aportando preguntas que fuercen a razonar. En ocasiones, resulta operativo que aquél dirija una discusión que provoque el desarrollo de habilidades tales como la «M-Capacity", el proceso y transformación de datos en varias direcciones, la segregación de información relevante, Ia organización de variables para la toma de decisiones, la independencia de campo y demás variables relacionadas con el pensamiento formal. 
4. Estimular a los alumnos con tendencia a una «laboriosidad horizontaly (simple retención cuantitativa de información) al reto que supone intentar superar la «dificultad verticals (razonamiento mediante procesos cualitativos superiores).

5. Procurar que exista un equilibrio en la valoración acadérnica del aprendizaje de procesos y de contenidos, aspectos que nunca pueden ser vistos como dicotómicos, sino como una realidad holística.

6. No deseo soslayar la dificultad de acometer la resoIución de problemas en un aula donde existe una manifiesta heterogeneidad del grado de desarrollo aptitudinal de los alumnos. Quizás pudiera ser útil la repetición de la resolución del problema, con una explicitación muy detallada, y que por consiguiente exija un menor desarrollo de las operaciones formales.

\section{REFERENCIAS BIBLIOGRÁFICAS}

AUSUBEL, D.P., NOVAK, J.D. Y HANESIAN, H., 1983. Psicología educativa: Un punto de vista cognoscitivo. Segunda Edición. (Editorial Trillas, México).

BODNER, G.M. y McMILLEN, T.L., 1986. Cognitive restrnturing as an early stage in problem solving, Journal of $R e$ search in Science Teaching, 23(8), pp. 727-737.

CHANDRAN, S., TREAGUST, D.F. y TOBIN, K., 1987. The role of cognitive factors in chemistry achievement, Journal of Research in Science Teaching, 24(2), pp. 145-160.

CUSAK, M., WIDEEN, M. y SHERWOOD, M. 1986. Teaching in secondary schools and its relationship to the development on formal operational thought, European Journal of Science Education, 8(1), pp. 95-106.

DRIVER, R. 1989. Student's conceptions the learning of science, International Journal of Science Education, $11(5)$ pp. $481-490$.

GARRETT, R.M., SATTERLY, D., GIL PÉREZ, D. y MARTIÍNEZ-TORREGROSA, J., 1990. Turning exercises into problems: an experimental study with teachers in training, International Journal of Science Education, 12(1), pp. 1-12.

GIL, D. y CARRASCOSA, J., 1985. Science learning as a conceptual and methodological change, European Journal of Science Education, 7(3), pp. 231-236.

GIL, D., MARTÍNEZ-TORREGROSA, J. y SENENT, F., 1988. El fracaso en la resolución de problemas de Física: una investigación orientada por nuevos supuestos, Enseñanza de las Ciencias, 6(2), pp. 131-146.

HAYES, J., 1980. The Complete Problem Solver. (Franklin Institute: Philadelphia).

KRAMERS-PALS, H. y PILOT, A., 1988. Solving quantitative problems: guidelines for teaching derived from research, International Journal of Science Education, 10(5), pp. 511-522.
7. Respecto a las actitudes de los alumnos, siempre será más motivador un problema relacionado con la vida real que aquél que está desconectado de los intereses de Ios alumnos.

Deseo finalizar este trabajo destacando una idea que subyace en el mismo -convicción apoyada por la investigación (Pomés 1986)-. Es que el continuo intento de provocar una tensión entre lo que se sabe y lo que demanda el problema resulta enriquecedor para el discente; dicho de otro modo, forzar a los alumnos a razonar al límite de sus potencialidades intelectuales, a pesar de ser tarea ardua, puede convertirse en instrumento decisivo para ese desarrollo cognitivo si está precedido por una actitud favorable (p.e. el desafío motivador de vencer las propias limitaciones).

LAWSON, J.E., 1985. A review of research on formal reasoning and science teaching, Journal of Research in Science Teaching, 22(7), pp. 569-617.

MORRIS, J.E., LAZONBY, J.N, y WADDINGTON, D.J., 1982. Calculations Involving the Mole Concept, an Enyuiry. into the difficulties Experienced by pupils, Chemical Education Resarch: Implications for Teaching. (Report of a Symposium). The Royal Society of Chemistry, London.

NIAZ, M., 1988: Manipulation of M-Demand of chemistry problems and its effect on student performance: a neopiagetian study, Journal of Research in Science Teaching, 25(8), pp. 643-657.

NIAZ, M., 1989. The Relationship betwen M-Demand, Algorithms, Problem Solving: A Neopiagetian Analysis, Journal of Chemical Education, 66(5), pp. 422-424.

POMÉS, J., 1986. Evaluación de una Química preuniversitaria. (Tesis doctoral). (Servicio de Publicaciones de la Universidad del País Vasco)

PASCUAL-LEONE, J., GOODMAN, D., AMMON, P. y SUBELMAN, I., 1978. Piagetian theory neo-Piagentian analysis as psychological guides in education. Contenido en Knowledge development. (J.M. Gallagher ed., Plenum: New York).

POOLE, R.L., 1989. Teaching Stoichiometry: A Two Cycle Approach, Journal of Chemical Education, 66(1), pp. 57-58.

POZO, J.I., 1989. Teorías cognitivas del aprendizaje. (Morata: Madrid).

SELVARATNAM, M. y FRAZER, M.J., 1982. Problem Solving in Chemistry. (Heinemann Educational Books: London).

SHAYER, M. y ADEY, $P_{+}, 1981$. Towards a science of science teaching. (Heinemann, London). 